\title{
Heart Rate Variability in Cancer-Related Fatigue
}

B Brady1,2, M Barrett', MJ Kennedy3,4, S Cuffe ${ }^{3}$, RT Murphy33, D Walsh1,2,4

1.Our Lady's Hospice \& Care Services 2.University College Dublin 3.St. James's Hospital 4.Trinity College Dublin

Background

- Cancer-Related Fatigue (CRF) is a common, high-impact symptom

- Pathophysiology poorly understood

- Hypothesis: Cardiovascular autonomic nervous system (CV ANS) dysfunction contributes to CRF

- Heart rate variability (HRV) is a noninvasive method of measuring CV ANS function

- HRV reported as root mean square of successive differences (RMSSD) and standard deviation of the NN (R-R) intervals (SDNN).

- SDNN represents total variability lower numbers indicate loss of dynamic response

\section{Outcome Measures}

FATIGUE

- BFI

- Hand grip strength

- Timed Up and Go (TUG)

- Sit to Stand (STS)

\section{ANS FUNCTION}

- HRV

- 5 mins spontaneous breathing

-5 mins paced breathing
Aim

Investigate CRF and CV ANS function in solid tumours

Methods

- Prospective observational study

- Consecutive, treatment naïve, oncology outpatients

- Exclusion: previous cancer, known cardiovascular disease

- Participants identified as 'fatigued' based on brief fatigue inventory (BFI) score of $\geq 3$

\section{Results}

$\mathrm{N}=10 ; 6$ ?

Age: Median 54 (range 40-73)

Cancer type:

- Breast 6

- Oesophageal 4

Loco-regional disease: 3

BFI: Median 1.25 (range 0-5.9)

BFI $\geq 3: 3 / 10(30 \%)$

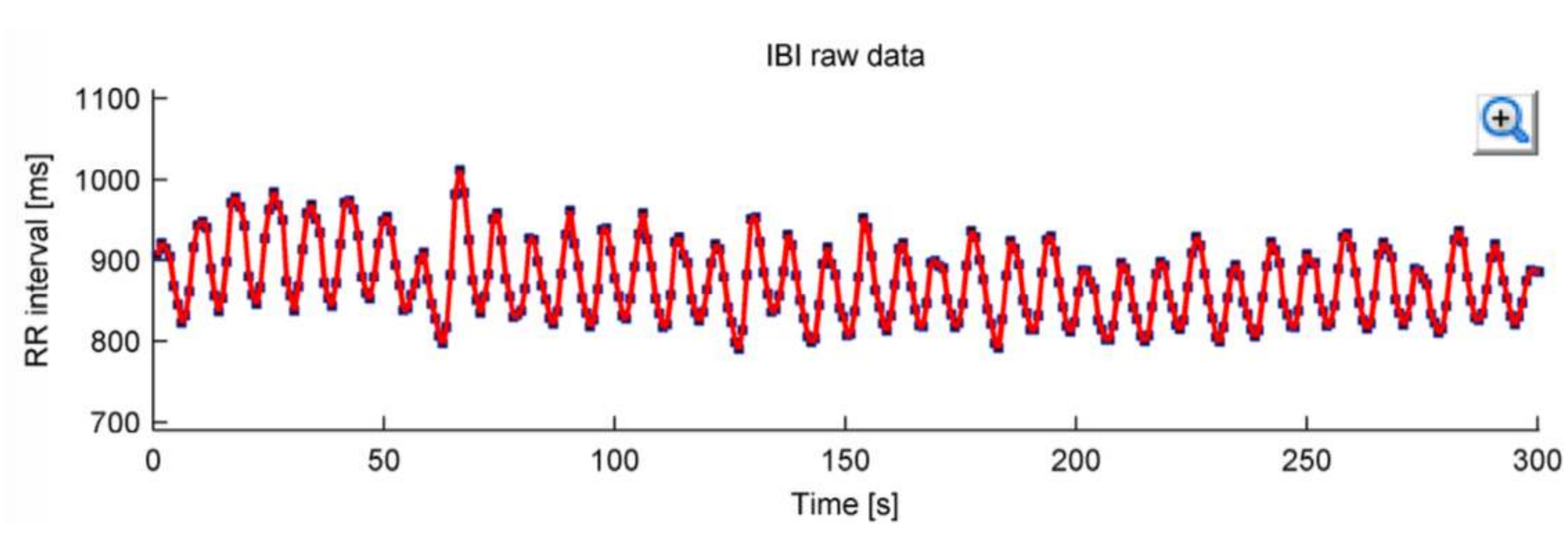

Sample HRV Analysis

\begin{tabular}{|c|c|c|}
\hline & $\begin{array}{r}\text { Fatigued } \\
\text { Median (range) } \\
N=3\end{array}$ & $\begin{array}{r}\text { Non-Fatigued } \\
\text { Median (range) } \\
N=7\end{array}$ \\
\hline Grip strength (kg force) & $23(18-33)$ & $35(14-48)$ \\
\hline TUG (s) & $8(7-8)$ & $7(6-9)$ \\
\hline STS (number in 30s) & $13(9-16)$ & $13(11-18)$ \\
\hline RMSSD, spontaneous & $16(6-25)$ & $18(11-79)$ \\
\hline RMSSD, paced & $18(8-71)$ & $23(17-86)$ \\
\hline SDNN, spontaneous & $22(18-27)$ & $30(17-124)$ \\
\hline SDNN, paced & $30(17-96)$ & $45(28-121)$ \\
\hline
\end{tabular}

\section{Conclusions}

- Significant proportion fatigued pre-treatment

- Indicators of CV ANS dysfunction present:

- Lower SDNN in fatigued participants

- Feasible study; tests well tolerated 